\title{
Buffered 1\% Lidocaine With Epinephrine Can Be as Effective as Nonbuffered 2\% Lidocaine With Epinephrine for Maxillary Field Block
}

\author{
James A. Phero, DDS, MD, ${ }^{*}$ Victor T. Warren, BS, $\dagger$ Anson G. Fisher, BA, $\neq$ \\ Eric M. Rivera, DDS, $\S$ Pooja T. Saba, BSPH, // Glenn Reside, DDS, $\rrbracket$ \\ Ceib Phillips, $\mathrm{PbD}, \mathrm{MPH}$, and Raymond P. White, Jr, DDS, PhD **
}

Purpose: Buffering local anesthetics with epinephrine (Epi) offers clinicians options not often considered. This study assessed outcomes for pulpal anesthesia, pain on injection, and time to midface numbness for buffered 1\% lidocaine with 1:100,000 Epi versus nonbuffered 2\% lidocaine with 1:100,000 Epi.

Materials and Methods: In this trial with a randomized, crossover design, buffered $1 \%$ lidocaine was compared with nonbuffered $2 \%$ lidocaine. Subjects were adult volunteers who served as their own controls. The predictor variables were alternate drug formulations. The outcome variables were subjects' responses to cold and electric pulp testing (EPT) stimulation of the maxillary first molar and canine, pain levels during the injection, and time to midface numbness. After maxillary field blocks with $40 \mathrm{mg}$ of buffered lidocaine or $80 \mathrm{mg}$ of nonbuffered lidocaine, subjects reported pain on injection and responses of the maxillary first molar and canine after cold and EPT stimulation. Teeth were tested before field block and at 30-minute intervals until a positive response was detected. Two weeks later, subjects were tested with the alternate drug combinations. For all outcome variables, assessment of treatment difference, calculated as $1 \%$ buffered minus $2 \%$ nonbuffered, was performed with the Wilcoxon rank sum test with significance at $P<.05$.

Results: More of the 24 subjects were women and Caucasian. The median age was 23.5 years (interquartile range, 21, 25 years), and the median body weight was $155 \mathrm{lb}$ (interquartile range, 128.5, 176.5 lb). Pain levels during the injection were significantly lower for $1 \%$ buffered lidocaine, with $P=.04$. Times to response after injection were not significantly different between the 2 drug formulations for the cold test on a molar, with $P=.08$, or the cold test on a canine, with $P=.22$. However, times to response were significantly longer for nonbuffered drugs for EPT on the molar and canine, both with $P=.01$.

Conclusions: Buffering 1\% lidocaine with 1:100,000 Epi reduces the pain on injection with a maxillary field block and results in similar lengths of pulpal anesthesia tested with a cold stimulus as compared with nonbuffered 2\% lidocaine with 1:100,000 Epi.

(C) 2017 American Association of Oral and Maxillofacial Surgeons

J Oral Maxillofac Surg 75:2071-2075, 2017

Received from University of North Carolina, Chapel Hill, NC.

*Resident, Department of Oral and Maxillofacial Surgery, School of Dentistry.

$\dagger$ Dental Student, School of Dentistry.

$\ddagger$ Dental Student, School of Dentistry.

$\S$ J. B. Freedland Distinguished Associate Professor, Department of Endodontics, School of Dentistry.

||Doctoral Candidate, Department of Biostatistics, Gillings School of Public Health.

TClinical Associate Professor, Department of Oral and Maxillofacial Surgery, School of Dentistry.

\#Professor, Department of Orthodontics, School of Dentistry.
**Dalton L. McMichael Distinguished Professor, Department of Oral and Maxillofacial Surgery, School of Dentistry.

Conflict of Interest Disclosures: None of the authors have any relevant financial relationship(s) with a commercial interest.

Address correspondence and reprint requests to Dr White: School of Dentistry, University of North Carolina, Chapel Hill, NC 27599-7450; e-mail: ray_white@dentistry.unc.edu

Received December 62016

Accepted March 172017

(c) 2017 American Association of Oral and Maxillofacial Surgeons

0278-2391/17/30350-6

http://dx.doi.org/10.1016/i.joms.2017.03.032 
Commercially available local anesthetic drugs formulated with epinephrine (Epi) have a low $\mathrm{pH}$ to prolong shelf life, usually around $\mathrm{pH} 3.5$. After the drug is injected, it is buffered by tissue fluid to a more neutral $\mathrm{pH}$. Because only the unionized form of the drug penetrates the targeted nerve membrane, more of the active drug is available in the unionized form when the $\mathrm{pH}$ of the injected local anesthetic solution is closer to the drug's $\mathrm{p} K_{\mathrm{a}} \cdot{ }^{1}$ The $\mathrm{p} K_{\mathrm{a}}$ is the $\mathrm{pH}$ when the unionized and ionized molecules of the drug are equal. For lidocaine, the $\mathrm{p} K_{\mathrm{a}}$ is approximately 8.0 with minor variation dependent on temperature. The drug injected at a neutral $\mathrm{pH}$ eliminates the time lag for buffering by tissue fluid, making the maximum unionized form of the anesthetic drug available at the site of injection immediately while retaining the desired qualities of the Epi.

Because more of the lidocaine in the unionized form is in proximity to the targeted nerve fibers immediately when the lidocaine with Epi is buffered, it is plausible that a lower drug dosage might be effective. Warren et $\mathrm{al}^{2}$ have reported that buffered $1 \%$ lidocaine with 1:100,000 Epi for a mandibular nerve block has a comparable clinical effect, in terms of duration of pulpal anesthesia, to commercially available nonbuffered 2\% lidocaine with Epi. In addition, $70 \%$ of subjects reported significantly lower pain scores after receiving buffered $1 \%$ lidocaine as compared with nonbuffered $2 \%$ lidocaine, with $P<.01$.

The purpose of this study with a crossover design, with each participant serving as his or her own control, was to assess clinical outcomes: pulpal response to cold and electric pulp testing (EPT) on the maxillary first molar and canine after a field block with buffered $1 \%$ lidocaine with 1:100,000 Epi as compared with nonbuffered 2\% lidocaine with 1:100,000 Epi. In addition, data were collected to substantiate previous reports of less pain during the injection and time to numbness after a field block in the skin and after a mandibular nerve block with buffered 1\% lidocaine with 1:100,000 Epi as compared with the $2 \%$ nonbuffered drug. ${ }^{2-9}$ We hypothesized that no differences in the outcomes studied would be detected when comparing the 2 drug formulations. The specific aims included determining subjects' reported differences between drug formulations in perceived pain on injection and the length of time, in 30-minute intervals after injection, to a return to sensation tested by cold and EPT stimulation on the maxillary first molar and canine.

\section{Materials and Methods}

\section{STUDY DESIGN AND SAMPLE}

To address the research purpose, we designed and implemented an institutional review board-approved study with a crossover design. Each participant served as his or her own control. The recruited study population, 24 subjects, was composed of adult volunteers tested at the University of North Carolina Oral and Maxillofacial Surgery Clinic 2 weeks apart in May 2016. No treatment other than the study tests was rendered. To be included in the study sample, subjects had to meet the following criteria: healthy individuals with American Society of Anesthesiologists class I or II, aged between 20 and 30 years, no allergy to lidocaine, and no receipt of a local anesthetic in the previous month. Individuals were excluded if they did not speak English or did not live within 30 miles of the clinic.

\section{VARIABLES}

The predictor variables for the study were the alternate drug formulations: $4 \mathrm{~mL}$ of buffered $1 \%$ lidocaine ( $40 \mathrm{mg}$ ) with 1:100,000 Epi or $4 \mathrm{~mL}$ of nonbuffered $2 \%$ lidocaine $(80 \mathrm{mg})$ with 1:100,000 Epi. The syringe for the buffered drug had an added volume of $0.4 \mathrm{~mL}$ to compensate for the added bicarbonate solution. The outcome variables were subjects' subjective responses to cold and EPT stimulation of the maxillary first molar and canine pain during the injection and time until the midface was numb.

\section{DATA COLLECTION}

Subjects were randomized to receive injections intraorally 2 weeks apart for maxillary field blocks with the 2 drug formulations: buffered and nonbuffered. The buffering kit connected the vials containing the local anesthetic and bicarbonate with Luer components allowing each milliliter of buffered local anesthetic drug to be buffered in turn with $0.1 \mathrm{~mL}$ of bicarbonate (Anutra Medical, Research Triangle Park, NC). A detailed description of the buffering kit is provided on the manufacturer's Web site (AnutraMedical. com). Subjects and clinicians recording their responses were masked to the drugs administered.

A full-time surgery faculty member administered the maxillary field blocks. The surgeon was blinded to the drug administered during each clinical session. In week 1, each participant received a unilateral infiltration injection on the facial and palate to anesthetize the maxillary first molar and canine. Two weeks later, sufficient to exceed the washout period for the drug, the field block on the same side of the jaw involved the alternate local anesthetic combination.

Pulpal anesthesia-blockage of sensory nerves within the teeth-was assessed by subjective reports from subjects after a cold test and EPT were applied to the maxillary first molar and canine. Both tests were conducted at baseline and at 30-minute intervals until the baseline positive response to testing was attained. If subjects did not feel a sensation by 
120 minutes, they were recorded as having felt a positive sensation at some time greater than 120 minutes. Time increments were ranked as 1 (30 minutes) through 5 (>120 minutes) for analyses. Cold stimulation targeted unmyelinated C sensory nerve fibers, and EPT targeted myelinated A delta sensory nerve fibers. ${ }^{10-13}$

Assessment of pain level during the injection was reported by each participant using a 10-point Likerttype scale anchored by "no pain" and "worst pain imaginable." A timed assessment was performed for clinical onset of anesthesia by the participant reporting the time after injection to a numb midface.

\section{DATA ANALYSES}

The sample size of 24 subjects was chosen to meet limitations on budget and clinic availability. This sample was sufficient to provide data for bivariate analyses, assess whether a larger study is warranted, and provide estimates for sample size calculation for larger studies. For all outcome variables, an assessment of treatment difference, calculated as $1 \%$ buffered minus $2 \%$ nonbuffered, was performed with the Wilcoxon rank sum test with Proc NPAR1WAY (SAS, version 9.3; SAS Institute, Cary, NC). ${ }^{14}$ Statistical significance was set at $P<.05$ for all outcomes.

\section{Results}

Of the 24 subjects completing the study protocol, $58 \%$ were women; $58 \%$ were white, $17 \%$ were African America, 17\% were Asian, and 8\% were other ethnic designations (Table 1). The median age was 23.5 years (interquartile range [IQR], 21, 25 years), and the median body weight was $155 \mathrm{lb}$ (IQR, 128.5, $176.5 \mathrm{lb}$ ).

When drug formulation times were combined (48 scores), the median time to return of sensation after injection for the cold test on a molar was 90 minutes. The median difference in time to recovery of sensation between $2 \%$ nonbuffered and $1 \%$ buffered lidocaine was approximately 15 minutes (median rank, 0.5 time increments). Half of the subjects reported the same time or a longer time to a positive response to cold on the maxillary molar after buffered $1 \%$ lidocaine as compared with nonbuffered $2 \%$ lidocaine. Time to sensation return was not significantly different, on average, between the 2 drug formulations for the cold test on a molar, with $P=.08$.

When drug formulation times were combined (48 scores), the median time to return of sensation after injection for the cold test on a canine was approximately 75 minutes (median rank, 1.5 time increments). The median difference in time to recovery of sensation between $2 \%$ nonbuffered and $1 \%$ buffered lidocaine was 0 minutes for the cold test on a canine. Seventy-one percent of subjects reported the same

\begin{tabular}{|c|c|}
\hline & Data \\
\hline \multicolumn{2}{|l|}{ Gender, $n$} \\
\hline Male & $10(42 \%)$ \\
\hline Female & $14(58 \%)$ \\
\hline Median weight (IQR), lb & $155(128.5,176.5)$ \\
\hline Median age (IQR), yr & $23.5(21,25)$ \\
\hline \multicolumn{2}{|l|}{ Ethnicity or race, $\mathrm{n}$} \\
\hline White & $14(58 \%)$ \\
\hline African American & $4(17 \%)$ \\
\hline Asian & $4(17 \%)$ \\
\hline Other & $2(8 \%)$ \\
\hline
\end{tabular}

Abbreviation: IQR, interquartile range.

Phero et al. Maxillary Field Block Using Lidocaine. J Oral Maxillofac Surg 2017.

time or a longer time to a positive response to cold on the maxillary canine after buffered $1 \%$ lidocaine as compared with nonbuffered $2 \%$ lidocaine. Time to sensation return was not significantly different, on average, between the 2 drug formulations for the cold test on a canine, with $P=.22$.

For the EPT on a molar, the median difference in time to recovery of sensation between $2 \%$ nonbuffered lidocaine and $1 \%$ buffered lidocaine was 30 minutes (median, 1 time increment). Only $35 \%$ of subjects reported the same time or a longer time to a positive response to EPT on the maxillary molar after buffered $1 \%$ lidocaine as compared with nonbuffered $2 \%$ lidocaine. Time to sensation was significantly different, on average, in favor of nonbuffered $2 \%$ lidocaine between the 2 drug formulations for the EPT on a molar $(P=.01)$.

For the EPT on a canine, the median difference in time to recovery of sensation between $2 \%$ nonbuffered and $1 \%$ buffered lidocaine was 30 minutes (median, 1 time increment). Almost half the subjects (48\%) reported the same time or a longer time to a positive response to EPT on the maxillary canine after buffered $1 \%$ lidocaine as compared with nonbuffered $2 \%$ lidocaine. Time to sensation was significantly different, on average, in favor of nonbuffered $2 \%$ lidocaine for the EPT on a canine $(P=.01)$.

When drug formulation times were combined, the median score for pain level during the injection on the 10-point Likert-type scale for the 24 subjects ( 48 scores) was 4 (IQR, 2.5, 5). Pain levels were lower, on average, by 1 unit for $1 \%$ buffered lidocaine (IQR, $-2.5,+1)$. Of 24 subjects, $58 \%$ reported lower pain scores after buffered $1 \%$ lidocaine as compared with nonbuffered $2 \%$ lidocaine. The median difference in pain level during the injection between the 2 drug 
combinations significantly favored $1 \%$ buffered lidocaine, with $P=.04$.

When drug formulation times were combined, the median time to midface numbness for the 24 subjects (48 scores) was 2 minutes (IQR, 1, 6 minutes). Of 24 subjects, $58 \%$ reported a lower time to midface numbness after buffered $1 \%$ lidocaine as compared with nonbuffered $2 \%$ lidocaine. The median difference in time to midface numbness between the 2 drug formulations was -2.5 minutes (IQR, $-5.5,+1.5$ minutes; $P=.33$ ).

\section{Discussion}

The data we report suggest that buffering $1 \%$ lidocaine with 1:100,000 Epi just before a maxillary field block offers clinicians options not often considered. After testing of a maxillary first molar and canine a cold stimulus, no differences in time to recovery of sensation were recorded between the 2 drug combinations-buffered $1 \%$ lidocaine and nonbuffered $2 \%$ lidocaine. At least half of the subjects in the study reported that the duration of pulpal anesthesia after injection of buffered 1\% lidocaine with Epi was comparable with that of nonbuffered $2 \%$ lidocaine with Epi. A contrasting pattern resulted with electrical pulp stimulation (EPT) of the first molar and canine. Significant differences were detected with EPT in favor of nonbuffered 2\% lidocaine with Epi.

Our data also suggest that injection of buffered 1\% lidocaine with Epi for a maxillary field block was significantly less painful than injection of the nonbuffered $2 \%$ drug. Although $58 \%$ of subjects reported a lower time to midface numbness after buffered $1 \%$ lidocaine as compared with nonbuffered $2 \%$ lidocaine, the median time to midface numbness was less predictable and not significantly different for the 2 drugs studied.

Are the differences we report for the cold test and EPT plausible even though the outcomes contrast with those reported for a mandibular nerve block? A review by Lin and Chandler $^{10}$ suggests an explanation. The response to cold stimulation is mediated by unmyelinated $\mathrm{C}$ sensory nerve fibers located throughout the body of the pulp. These nerve fibers are associated with dull, lingering pain. The myelinated A delta sensory nerve fibers terminate at the pulpal-dentin junction relatively closer to the tooth surface. These fibers are associated with sharp, acute pain. On the basis of their terminal anatomic structure and location in the pulp, the A delta sensory fibers have a lower threshold for a response than the C sensory fibers. With a mandibular nerve block, the greatest volume of injected local anesthetic is concentrated in one anatomic region, close to the nerve trunk. With a maxillary field block, as the term "field" suggests, the injected local anesthetic is spread across a wider anatomic area as compared with a nerve block. Further investigation is needed to determine whether higher dosages of buffered 1\% lidocaine with Epi would produce similar outcomes with a maxillary field block as reported for a mandibular nerve block.

The data suggesting less pain on injection for a maxillary field block are similar to data in the literature regarding skin. Significantly less pain on injection of buffered 1\% lidocaine with Epi as compared with the nonbuffered drug has been reported for cosmetic procedures with a field block in skin, particularly for sensitive anatomic sites. For example, Lee et $\mathrm{al}^{5}$ reported less pain with buffered lidocaine as compared with nonbuffered in surgery of the hand, a traditionally painful anatomic site for injections. Masters ${ }^{4}$ found similar results with buffered local anesthetic administered for plastic surgery procedures; half of the procedures were performed on the face.

Clinicians should consider that the data we report have limitations. Although the experimental design compared outcomes for the same participant and we did not detect a "period effect," all data required subjective responses. Responses to stimulation of the test molar and canine are commonly used in dental practice, applied clinically to assess "tooth vitality." The assumption is that a positive response is accompanied by an adequate pulpal blood supply and a negative response is associated with necrotic pulp. Our method to assess blockage of sensory nerve responses by local anesthetics has been applied in experimental protocols by other investigators as early as the mid 20th century, but the tests were not originally designed for this application. ${ }^{10}$

It was clear that the local anesthetic buffered just before injection was associated with significantly less pain during the injection for a maxillary field block, a benefit to patients. However, reported pain levels were low, with an average of 4 on a 10-point Likert-type scale, irrespective of the test drug. Clinicians must weigh these potential benefits in planning short, simple procedures accomplished with local anesthesia alone as compared with longer, complex procedures with local anesthesia and sedation. The subjects studied were all young, healthy adults. Potential patients with a more compromised health status or a long history of complex dental problems may report different responses.

In summary, reducing drug dosages while retaining the benefits of effective responses to local anesthetics should be explored further. Clear benefits could accrue to pediatric patients whose lidocaine drug dosages and treatment are now limited by caution not to exceed maximum blood lidocaine levels based on body weight. Other patients may also benefit, particularly those with compromised liver function. Similar studies should be conducted for outcomes with other 
local anesthetics. For example, buffered $2 \%$ articaine may be as effective as the $4 \%$ drug, minimizing or eliminating the chance of sensory nerve deficit after a mandibular nerve block with $4 \%$ articaine while retaining the positive properties of the drug, including ready diffusion in tissue and a short elimination half-life in the bloodstream.

\section{Acknowledgment}

The National Institutes of Health Clinical Trials Registration number is NCT02747186. The authors acknowledge and appreciate Anutra Medical for supplying the buffering kit for lidocaine.

\section{Press Release}

This article's Press Release can be found, in the online version, at http://dx.doi.org/10.1016/j.joms. 2017.03.032.

\section{References}

1. Strichartz GR, Sanchez V, Arthur GR, et al: Fundamental properties of local anesthetics. II. Measured octanol: Buffer partition coefficients and pKa values of clinically useful drugs. Anesth Analg 71:158, 1990

2. Warren VT, Fisher AG, Rivera E, et al: Buffered $1 \%$ lidocaine with epinephrine effective for mandibular nerve block. J Oral Maxillofacial Surg 75:1363, 2017
3. Christoph RA, Buchanan L, Begalia K, Schwartz S: Pain reduction in local anesthetic administration: Through $\mathrm{pH}$ buffering. Ann Emerg Med 17:117, 1988

4. Masters JE: Randomised control trial of $\mathrm{pH}$ buffered lignocaine with adrenaline in outpatient operations. Br J Plast Surg 51: 385,1998

5. Lee HJ, Cho YJ, Gong HS, et al: The effect of buffered lidocaine in local anesthesia: A prospective, randomized, double-blind study. J Hand Surg Am 38:971, 2013

6. Burns CA, Ferris G, Feng C, et al: Decreasing the pain of local anesthesia: A prospective, double-blind comparison of buffered, premixed $1 \%$ lidocaine with epinephrine versus $1 \%$ freshly mixed with epinephrine. J Am Acad Dermatol 54:128, 2006

7. Malamed SF: Buffering local anesthetics in dentistry. Pulse 44:6, 2011

8. Malamed SF, Tavana S, Falkel M: Faster onset and more comfortable injection with alkalinized $2 \%$ lidocaine with epinephrine 1:100,000. Compend Contin Educ Dent 34(Spec No 1):10, 2013

9. Kashyap VM, Desai R, Reddy PB, Menon S: Effect of alkalinisation of lignocaine for intraoral nerve block on pain during injection, and speed on onset of anaesthesia. Br J Oral Maxillofac Surg 49: $\mathrm{e} 72,2011$

10. Lin J, Chandler NP: Electric pulp testing: A review. Int Endod J $41: 1,2008$

11. Dreven LJ, Reader A, Beck FM, et al: An evaluation of an electric pulp tester as a measure of analgesia in human vital teeth. J Endod 13:233, 1987

12. Modaresi J, Ali Mozayeni M, Dianat O: Comparing the quality of anaesthesia in normal and inflamed teeth by pulp testing. Aust Endod J 31:120, 2005

13. Chen E, Abbott PV: Evaluation of accuracy, reliability, and repeatability of five dental pulp tests. J Endod 37:1619, 2011

14. Simpson PM, Hamer RM, Lensing S: Cross Crossover Studies Off Your List. Presented at the SAS Users Group International Conference, Miami Beach, FL, April 11-14, 1999. Available at: http://www2.sas.com/proceedings/sugi24/Posters/p221-24.pdf. Accessed August 1, 2016 